\title{
Micro XRF Element Maps Are A New Method Of Detecting Elements At Lower Concentrations Than The Electron Beam Produced Corollary: A Garnet Schist Example
}

\author{
C.S. Schwandt
}

McCrone Associates, Inc., 850 Pasquinelli Drive, Westmont, IL 60559-5539

Recent improvements in capillary fiber optics appropriate for the transmission of X-rays now permit the focusing of X-rays into a reasonably bright beam only a few tens of micrometers in diameter. Attaching such a source onto a scanning electron microscope (SEM), that already has a functional energy dispersive X-ray spectrometry system (EDS), offers distinct advantages. These include that there is typically a computer controlled motorized stage, the sample chamber by design already shields the investigator from unintended X-ray exposure, and samples can be examined using electron beam excitation, X-ray beam excitation, or both simultaneously.

The primary reason for using an X-ray beam rather than an electron beam for producing characteristic X-ray spectra is that the background in the spectra is significantly lower; 100 to 1000 times lower depending on the element [1]. The lack of beam electron - sample atom interactions means the classic EDS Bremsstrahlung background is not present to interfere with the peak to background ratio of elements in the sample; therefore one should theoretically be able to detect elements in a sample at lower concentrations.

This is demonstrated with element maps shown in Figure 1. A garnet with surrounding schist matrix from the Black Hills, South Dakota, was mapped using a micro X-ray source (IXRF Systems X-Beam) mounted on a JEOL SEM. The garnet had previously been analyzed for rare earth element (REE) zoning using secondary ion mass spectrometry (SIMS) [2]. The SIMS analyses demonstrate that the garnets of the schist recorded complex trace element zoning at concentrations generally below electron microprobe analysis detection limits. The SIMS maximum concentrations in one garnet are presented in Table 1. Importantly, several factors complicated earlier attempts to understand the distribution of the trace elements in the schist as a whole. Trace elements, especially REE, fit into the crystal structures of the matrix minerals (e.g. micas and feldspars) at much lower concentrations than the garnet structure. The distribution within garnets is extremely complex. Additional REE-rich phases exist. Trace elements might be mobile by more than one pathway. Therefore, obtaining trace element distribution maps is critical to addressing these issues. However, until now acquisition of these data was unrealistic due to limits of detection and analytical cost.

Inspection of the X-beam element maps (Fig. 1) for spectral overlap is especially critical as characteristic energies of many trace elements of interest occur near the characteristic energies of major element constituents. Overlap issues are partially addressed by selecting regions of interest for each element, which are relatively narrow so that one considers predominantly peak intensities and not tail intensities. The next step for demonstrating the presence of actual trace element intensity changes is to examine a sum map normalized to the major peak. With maps one can visualize this quickly. For example it is easy to see that some of the REE spectra which have energies near Fe actually show REE variations which are not mere weak copies of the Fe map. While this simple approximation shows relative concentration differences, it is more difficult to quantify the values, because the extent of interference and matrix changes are not taken into 
account. However, a crude estimate is constructed by normalizing the REE map and applying a known ratio, for example $\mathrm{Nd} / \mathrm{Fe}$, to the normalized map. Figure 2 reveals a concentration in the mica phase lower than in garnet, as well as $\mathrm{Nd}$-rich phase higher than in garnet.

Quantitative data could be extracted if an X-beam were mounted either on an electron microprobe or a SEM with a wavelength dispersive spectrometer system (WDS) attached. However, without WDS, quantitative results could be achieved using "sample-like" standards with a range of concentrations for each phase to construct calibration curves for each phase. Even without quantification, being able to visualize the distribution of trace elements is a huge step forward.
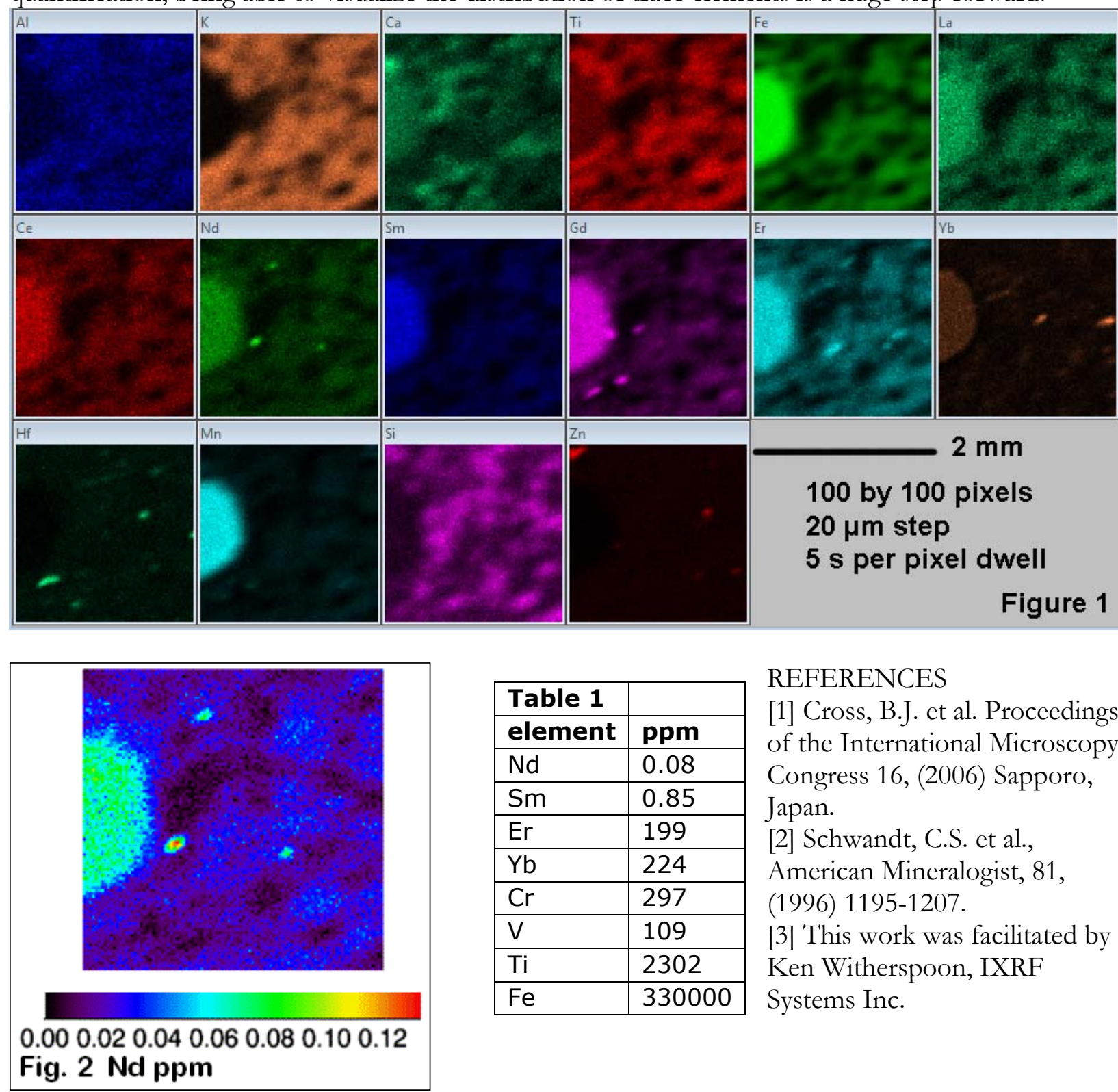

\begin{tabular}{|l|l|}
\hline Table 1 & \\
\hline element & ppm \\
\hline $\mathrm{Nd}$ & 0.08 \\
\hline $\mathrm{Sm}$ & 0.85 \\
\hline $\mathrm{Er}$ & 199 \\
\hline $\mathrm{Yb}$ & 224 \\
\hline $\mathrm{Cr}$ & 297 \\
\hline $\mathrm{V}$ & 109 \\
\hline $\mathrm{Ti}$ & 2302 \\
\hline $\mathrm{Fe}$ & 330000 \\
\hline
\end{tabular}

\section{REFERENCES}

[1] Cross, B.J. et al. Proceedings of the International Microscopy Congress 16, (2006) Sapporo, Japan.

[2] Schwandt, C.S. et al., American Mineralogist, 81, (1996) 1195-1207.

[3] This work was facilitated by Ken Witherspoon, IXRF Systems Inc. 